\title{
SABERES Y COMPORTAMIENTOS ÉTICOS NECESARIOS EN LA EDUCACIÓN DEL FUTURO
}

Elvia Marveya Villalobos Torres

\section{RESUMEN}

ESTE ARTículo BUSCA MOSTRAR LA NECESIDAD DE VALORAR Y TENER presentes los saberes y comportamientos éticos fundamentales para una educación del futuro, puesto que la realidad, hoy, está inmersa en el desamollo tecnológico desmedido y en procesos de globalización que invaden y modifican la vida. Los procesos educativos no deben perderde vista la formación personal.

\section{ABST RACT}

Since reality nowadays is immerse in an immoderate technical developmentand in globalization processes that invade and modify life, this article intends to show the need to value and set in mind the ethical knowledge and behavior that are vital for the future education system. The educative processes should notforgetabout the personal formation.

\section{INTRODUCCIÓN}

Todo proceso educativo implica intelecto y voluntad. Intentar su separación seńa un emor porque no se trata de formar buenos cientíicos, sino buenas personas. Deseo y saber; voluntad e intelecto. No puede producirse uno sin el otro.

Voluntad desligada del intelecto, será sólo la configuración final de las fuerzas emotivas e instintivas. Una concepción fraccionada de la voluntad, representa un problema para la educación: carece de continuidad en los procesos, pero sobre todo de una falta de unidad personal. 
Es necesania la unidad de las dos capacidades específicamente humanas: intelecto y voluntad (ésta como elemento esencial del sabery de una educación crecientemente perfectiva).

La búsqueda serena de la verdad requiere que la enseñanza educativa se dirija a la razón completa y plena; a la razón que apetece saber, al entendimiento que comprende, movido porel apetito racional, porla voluntad como deseo y como supremo deseo del saber.

El intelecto actúa unido a la voluntad. Y cuando existe separación se genera la crisis. La voluntad es la gran olvidada de la cultura contemporánea y ese descuido se paga en forma de crisis personal y social. El reto es conocer con la voluntad; la educación nos guía a la adquisición de la verdad.

Los saberes y comportamientos éticos necesarios en la educación del futuro exigen, en primera instancia, la presencia total, integral, de la enseñanza formativa, de la plenitud de la persona.

\section{SABERES Y COMPORTAMIENTOS ÉTICOS}

Estamos en el alba del siglo XXI, de la tercera revolución industrial y de las nuevas formas de mundialización que la acompañan, se transforman nuestras sociedades de manera radical y nos invitan a enfrentarun gran número de desafíos inéditos. El mundo y la aventura humana aparecen más ricos en potencialidades, más complejos, más interdependientes pero sin duda, también, más inciertos que nunca.

Hoy es necesario quitar toda esa fuerza centrífuga, provocada por la aceleración considerable del nitmo de las evoluciones, que el progreso al que nos referimos puede amenazar, quitando el registro de lo humano.

Es fundamental entender las relaciones entre las cosas, saberreleer las ideas, darles coherencia y pertinencia; esto deviene en uno de los actos esenciales. Es necesario explorar esta riqueza, esta diversidad, esta interdependencia, esta incertidumbre inherente del futuro.

El trabajo de reflexión prospectiva, transdisciplinaria, es indispensable para dar sentido a la acción. La tarea es aprendera descifrar el 
concierto de los signos del futuro y traduciros en visiones compartidas y en proyectos comunes. Esto es lo que significa vivirjuntos.

Daniel Cohen ${ }^{1}$ afirma que el principio de la tercera revolución industrial está fundado sobre el desamollo de la informática que permite a cada uno ser el motorinmóvil de una infinidad de desplazamientos virtuales.

Edgar Morín ${ }^{2}$ pregona la necesidad de una reforma del pensamiento y la educación. Goéry Delacóte ${ }^{3}$ ofrece una nueva visión de la enseñanza que será practicada en el futuro, teniendo un mejor condicionamiento de la cognición humana. Según él, pasaremos muy pronto de una cultura del aprendizaje a una cultura del «cómo aprender» Roberto Carneiro ${ }^{4}$ anuncia la emergencia de una sociedad cognitiva.

¿Cómo lograr la reforma del pensamiento y de la educación?Para saberlo se requiere sensibilizamos ante la contradicción que actualmente vivimos: de una parte están los problemas globales, interdependientes y planetarios; de otra, nuestro modo de conocer resulta cada vez más fragmentado, parcelado. Es éste es el principal desafío lanzado al conocimiento, a la educación y al pensamiento; identificado desde el siglo XX, ese problema se acentuará y deberá serresuelto en este siglo.

Hoy nuestro pensamiento debe sercapaz de aprehenderel mundo globalmente y en sus partes.

Necesitamos ser capaces de contextualizar lo singular, lo particulary lo local y, a la vez, de concretar lo global. Es decir, incluirlo en relación con las partes. Un ejemplo: el comportamiento ético de un hijo repercute en la estructura global de la familia y por ende de la sociedad. En este contexto, el griego Margalit 5 afirma que sólo tendremos sociedades decentes, cuando haya instituciones decentes y porende personas decentes.

Es necesario elaborar un modo de reflexión que sea capaz de dar cuenta de la complejidad del mundo actual. De otra forma estaremos ciegos o miopes en nuestra actitud frente al mundo. 
Se requiere llevar al cabo la reforma del pensamiento y ésta es inseparable de la reforma educativa.

¿Cuáles son los grandes temas ${ }^{6}$, conocimiento o saberes que deben ser fundamentales en nuestra enseñanza?En primer lugar, es necesario rescatar el principio de MONTAIGNE del siglo XVI:

Más vale una cabeza bien hecha que bien llena?

La enseñanza no debe tender a la acumulación de los conocimientos, sino a su organización en función de ejes estratégicos esenciales. La noción sistémica nos abastece para regular los conocimientos

Yo quiero enseñarle la condición humana ${ }^{8}$,

afirmación de Jean-Jacques Rousseau en el «Emilio» Es necesario restablecer la relación entre el conocimiento científico y el de las humanidades.

Lo anterior implica enniquecer el currículum con el asesoramiento personal de los estudiantes, la introducción de actividades complementarias como parte del currículum habitual, un enfoque activo de la educación y la idea de que los alumnos deben aprendera ser responsables en un ambiente de libertad ${ }^{9}$. Esta idea de libertad responsable debe traducirse, inmediatamente, en una atención al desamollo de los comportamientos éticos o virtudes humanas, o hábitos buenos $^{10}$. En esencia, se trata de una orientación del comportamiento humano con la formación de actitudes, valores y normas ${ }^{11}$.

La educación en valores es un tema pedagógico candente que, por lo demás, lleva consigo no pocas dificultades teóricas y graves consecuencias educacionales, de no tomarse en cuenta. Hoy requerimos de un desarrollo tecnológico de punta, pero también de un desarrollo humano de punta.

Demandamos la participación estrecha con los padres de familia, 
protagonistas indiscutibles en todo lo referido a los aspectos educativos más íntimos.

En los centros educativos, enseñar la condición humana exige del ejercicio de comportamientos éticosy, en consecuencia, la necesidad de lograr la madurez humana como fin de la educación ${ }^{12}$.

Una justificación más para enseñarla condición humana, es considerar que la educación de los comportamientos éticos - como algo prioritario en los centros educativos- surge si se considera el perfeccionamiento como el fin de la educación. Para el pensamiento clásico, la definición de felicidad es aquélla según la cual una persona es feliz si consigue desarrollar todo lo que es de acuerdo con la naturaleza humana.

La inteligencia del hombre le permite descubrir lo bueno para legar, en un estadio superiory más maduro, a un reconocimiento, aprecio y disfrute sereno de la vida ${ }^{13}$.

Al enseñar la condición humana, es necesario distinguir qué cosas o placeres constituyen una felicidad cada vez más estable, porque sólo a eso se puede llamar felicidad: a lo que colma y dura más.

El comportamiento ético o virtud es consecuencia de habercomprendido el interés en el valor que representa, para lograr una atención congruente y autónoma.

Enseñar la condición humana exige la formación en comportamientos éticos o virtudes humanas, que realimentan el entendimiento y la voluntad de tres modos principales: la firmeza, porque reafirma en la persona lo que está haciendo; la prontitud, al crearuna capacidad con másfacilidad; y porúltimo, obrar a gusto, que permite conocer en parte la felicidad, así como pensar, sentiry actuar con satisfacción, es decir con coherencia de vida.

$$
\text { Yo quiero enseñarle a vivir. }
$$

En el «Emilio», Jean-Jacques Rousseau afirma: ‘yo quiero enseñarle a vivin» En efecto, el aprendizaje no toma en cuenta sólo los 
saberes, las técnicas y los modos de hacer. debe interesarse también en las relaciones con el otro y consigo mismo.

Es importante reconciliar la cultura científica y la cultura de las humanidades. Quintana Cabanas afirma que la educación consiste básicamente en enseñar a vivir la vida ${ }^{14}$.

\section{Formarciudadanos.}

Los ciudadanos deben estarconscientes de su doble pertenencia: nacional y planetaria. La inteligencia consciente nos lleva a concebimos en la tierra-patria ${ }^{15}$.

La reforma del pensamiento no constituye un lenguaje intelectual; responde a una necesidad vital, es uno de los componentes para salvaguardarla humanidad.

Debemos concebimos como ciudadanos planetanios, con responsabilidad local y global. Hoy, la educación es un suceso y el desamollo del sistema educativo constituirá uno de los principales desafíos del siglo XXI.

Los cinco ángulos a considerarpara reformarla educación son: el desarmollo del sistema educativo; los saberes; el tiempo; el espacio y la cultura.

1. El desarrollo del sistema educativo. La formación del maestro como creadorde la nueva cultura, será el eje axial en el desamollo del sistema educativo. El cambio principal que afectará al sistema educativo será la transferencia de la responsabilidad del aprendizaje del maestro hacia el alumno. La función del maestro 〈evolucionará»del ser abastecedor de conocimientos, a veces muy perfeccionados, a serel encargado de «guiar»los aprendizajes.

El maestro no se reducirá más a compilarinformación de fuentes diversas; él será un cierto tipo de modelo cognitivo en acto, capaz de manifestar de manera explícita los mecanismos cognitivos a los cuales las personas no se saben generalmente confrontadas, puesto que ellas no aprecian los resultados. Por otro lado, el desamollo del sistema edu- 
cativo exigirá una mayor inversión de todos los útiles didácticos para llevaral cabo la interactividad - con la multimedia educativa— - en cuanto a aprendizajes de informaciones y conocimientos que deben reformar la cognición humana mediante la presencia de redes.

Para el desamollo de los sistemas educativos, otro aspecto fundamental serála evaluación, que exigirá mayornigor. Ello se acentuará considerablemente, aunqueignoremos cómo proceder. Hoy esnecesanio crear una auténtica cultura de la evaluación» entendida como parte del proceso educativo que permite el perfeccionamiento del acto educativo, una cultura que valore los aspectos positivos, que aprecie los avances.

2. El saber es otro aspecto fundamental. En el futuro, habrá una concepción más equilibrada del saber. Existirá mayor armonía entre los saberes puramente formales, los saberes de aplicación y los metasaberes. Accesibles en forma de productos, los saberes declarativos - según el téminino de los cognoscitivistas-, serán conjugados con los saberes procedimentales llamados a desarrollarse.

Hoy los saberes son transmitidos por la palabra y la escritura. El reto será participar de una nueva manera: multimedia e Internet. Las personas estarán estrechamente asociadas para la creación del sabery para la participación en el aprendizaje; se trata de la co-creación y de la co-participación de maestros y estudiantes ${ }^{16}$.

3. El tiempo para el aprendizaje disminuirá ligeramente gracias al mejor dominio de los mecanismos cognitivos que influirán, favorablemente, sobre la eficacia de las prácticas de aprendizaje.

La introducción de los saberes fundamentalesy prácticas sobre la cognición humana, reducirá los tiempos acordados para el aprendizaje. Será necesanio acostumbramos a un nuevo modo de intercambio en el cual no estamos entrenados.

Lo más importante será el aprendizaje a lo largo de toda la vida, aunque los objetivos y los valores aprendidos a los 2, a los 18 ó a los 50 años serán revisados en cada etapa.

4. El espacio. Se trata de una transformación profunda puesto que los espacios de aprendizaje serán muy dispersos, menos específicos 
y, porlo tanto, menos protegidos. Dejarán de serespacios o santuarios consagrados al saber, por el uso de la multimedia y la interactividad a distancia.

5. La cultura. En primer lugar es fundamental subrayar que, innegablemente, la cultura del aprendizaje se afectará por la interactividad (entendida en su sentido más amplio). Redesy útiles deberán adaptarse a esta nueva exigencia.

En segundo lugar, un mejor dominio de la cognición humana ${ }^{17}$ ejercerá sus efectos sobre la cultura del aprendizaje. El conjunto de los estudios realizados en este dominio subraya que la práctica cognitiva de las personas está hoy escondiday se hará explícita.

En tercer lugar, la organización de las instituciones educativas - en todos los niveles del sistema educativo - perderá probablemente su lugar fijo (hoy cerrado y nígido en los procesos de aprendizaje). Las modalidades de funcionamiento, las relaciones jerárquicasy la utilización de competencias deberán favorecer el surgimiento de instituciones de aprendizaje (learninginstitutions) aptas para afrontarel esfuerzo de laspersonas deseosas de aprender ${ }^{18}$.

En cuarto lugar, la cultura que impregnará esos cambios, dejará su naturaleza de «cultura del aprendizaje» para convertirse en «cultura para aprender»

¿Cuál será el esquema de la cultura para aprender?El desplazamiento desde el centro de los conocimientos hacia la penferia del saber vivo, del saber en movimiento. Tendrá lugar el cambio de lo local a lo nacional y del monopolio mediático - hoy detectado por las grandes potencias- hacia un polo múltiple, multinacional y multicultural.

Del mismo modo, se evolucionará de una práctica de lo opaco y del producto hacia una práctica de lo explícito. Se ponderarán los procesos, gracias a la puesta en evidencia del comportamiento mental, cultural y creativo del otro, que implica una confrontación, contradicción, conflicto verbal, diálogo, movimiento caótico del pensamiento; porello es indispensable que prevalezcan los comportamien- 
tos éticos. Por último, predominará la adquisición de valores trascendentes ${ }^{19}$, jamás inmutables, que motiven el aprendizaje.

Al estudiante también le serán reasignadas las funciones: deja de serun consumidorpasivo, deja de aprender a matar el tiempo» sale de egociudadano y se convierte en ecociudadano ${ }^{20}$, en medio de una pedagogía basada en el hacer, que pone énfasis en la práctica y en el saber hacer. La pedagogía que implica al estudiante, es una pedagogía que lo involucra integralmente. En esa pedagogía de la autonomía y la autoevaluación, su papel es más activo y decisivo que nunca.

Es a él a quien toca creary construir activamente conocimientos significativos, es él quien organiza conocimientos a partir de indicaciones del profesor. Se convierte en un investigador, en un constructor que es, en gran medida, un constructor de su proceso de enseñanza-aprendizaje - depende de él, del interés particular que posea- . Las clases se subordinan a ese interés para que, efectivamente, se sienta motivado en el trabajo que realiza. El estudiante se vuelve constructor activo, colaborador, experto, es el centro del trabajo, establece relaciones con el sabery con los conocimientos de su época.

El alumno desarrolla capacidades de apropiación profesional de información y de saberes; asume, en ciertos puntos, el papel de experto y gestor de sus propias contradicciones. Cada estudiante es, a su vez, el nudo de una comunicación y una fuente de producción de conocimientos en el aula. Además, el alumno sigue un itineranio cognoscitivo individual y debe desarmollar gradualmente una capacidad metacognitiva ${ }^{21}$ (es decir, reflexionar sobre su propio proceso de cómo conoce; no sólo aprende conocimientos sino también reflexiona sobre sus propios conocimientos y así planifica mejor, evalúa mejor). Serán un profesory un estudiante estratégicos. 


\section{CONCLUSIÓN}

1. Desarrollo cognoscitivo y desarrollo moral, de forma paralela, se constituyen en los saberesy comportamientos éticos necesarios en la educación del futuro.

2. La educación como proceso, tiene como objetivo terminal: aprendera vivir en una sociedad plural, donde los valores trascendentales sean el vínculo que cohesione la tarea educativa.

3. Para los profesionales de la educación nos debe quedarclaro que todo progreso procede de una liberación de fuerzas creativas.

\section{REFERENCIAS BIBLIOGRÁFICAS}

${ }^{1}$ UNESCO., «es nauvelles frontières de l'éducation»in Les clés du XXIe siècle., Ed. Seuil., Paris., 2000., p.269.

${ }^{2}$ MORIN, E., Réforme de la pensee etéducation au XXIe sieclesin UNESCO., Lesclésdu XXIesiecle., Ed. Seuil., Paris., 2000., p.271-275.

${ }^{3}$ DELACOTE, G., «Education a distance, nouvelles technologies et nouvelles methodes d'apprentissage»in UNESCO., Lesclés du XXIe siécle., Ed. Seuil., Paris., 2000., p.276-281.

${ }^{4}$ CARNEIRO, R, «'éducation pour tous tout au long de la vie et les nouveaux programmes»in Lesclésdu XXIe siécle., Ed. Seuil., Paris., 2000., p.282-286.

${ }^{5}$ MARGALT, A., La sociedad., p.15.

${ }^{6}$ Los presentamos como saberes, temas, problemas, conocimientos, pero en última instancia nos referimos a los aspectos que, por ellos mismos, nos permitan integrar las disciplinas existentes y estimular el desamollo de conocimiento que nos ayude a enfrentar los desafíos de nuestra vida individual, cultural y social.

${ }^{7}$ MORIN, E., La téte bien faite., p.23.

${ }^{8}$ La infancia no se conoce en absoluto. Cuanto más siguen las falsas ideas reinantes, más nos perdemos. Los más sabios se fijan en lo que conviene sabera los hombres, sin considerarlo que los niños son capaces de aprender. Buscan siempre al hombre en el niño sin pensar en lo que es antes de ser hombre. Éste es el estudio al que me he dedicado, a in de que, aunque 
todo mi método fuera quiménico y falso, siempre se pudiera sacarprovecho de mis observaciones. Puedo habeme equivocado completamente respecto a lo que hay qué hacer, pero creo que he examinado bien al sujeto sobre el que hay que actuar. Comenzad pues por estudiar mejor a vuestros alumnos, porque con seguridad no los conocéis en absoluto» [Rousseau., 1762., p.32]. Para enseñar la condición humana, Rousseau se fundamentó en la conducta de los niños, para actuarsobre ella y modificarla pormedio de la educación.

${ }^{9}$ ROUSSEAU, J.J., Emile ou del'Education., Libro I., p.79.

${ }^{10}$ Una tendencia fundamental en la educación moral y cívica contemporánea es su orientación al desamollo de la autonomía de losindividuos como valor preeminente. Esta orientación tiene su inspiración, en gran parte, en el pensamiento de Rousseau para quien la culminación de la libertad está precisamente en la autonomía que no supone ausencia de límites, sino aceptación voluntaria de ellos» NAVAL C., «a autonomía: fin último de la educación moral?»en Lengua, Literatura y Valores., p.141-154.

${ }^{11}$ El verdadero saber educativo (... ) es propiciar el cumplimiento de la norma, presentarla como referencia para la acción e ir gradualmente desechándola como última motivación en la formación moral. Esto se realiza promoviendo en el educando la reflexión sobre la acción realizada, sus motivos y también sus efectos» NAVALC., ¿a autonomía: fin último de la educación moral3»en Lengua, Literatura y Valores., p.141-154.

${ }^{12}$ El hombre es perfectible siempre en virtud de su propio actuar, dado que el perfeccionamiento humano no tiene techo; siempre se puede crecer y se puede fallar. Es así como el hombre es ético y es un ser social. A medida que el hombre realiza lo ético, sus tendencias son más fuertes, pero la fortaleza no es fuerza física, sino resistir la adversidad y ser flexible, es decir, encontrar la altemativa» NAVALC., «a autonomía: fin último de la educación moral?»en Lengua, Literatura y Valores., p.141-154.

${ }^{13}$ ¿a educación se nos presenta como el desarmollo del hombre bajo la acción consciente e inteligente de la voluntad; por eso sólo un sujeto libre, consciente e inteligente es susceptible de educación. De lo que resulta que la educación es un hecho peculiamente humano, y es el existirmismo del 
hombre» NAVALC., «La autonomía: fin último de la educación moral?» en Lengua, Literatura y Valores., p.141-154.

${ }^{14}$ QUINTANA CABANAS, J.M., Pedagogía Axiológica., p.13.

${ }^{15}$ Educar para la democracia, la ciudadanía, la diversidad, o si se prefiere, educar para la convivencia, es una necesidad apremiante en la sociedad actual, en el marco de las democracias liberales» NAVAL, C., «Es posible una continuidad entre hombre y sociedad?»en Revista Española dePedagogía., año XVII., mayo-agosto., 2000., No.216., p.275-290.

${ }^{16}$ MORIN, Edgar., Les sept savoirs..., p.36.

17

PROCESO COGNITIVO

\begin{tabular}{|c|c|c|c|c|}
\hline $\begin{array}{l}\text { Lo real: } \\
\text { El ente }\end{array}$ & $\begin{array}{l}\text { Sentidos } \\
\text { extemos: } \\
\text { Vista, oído, tacto, } \\
\text { gusto, olfato }\end{array}$ & $\begin{array}{l}\text { Sentidos intemos: } \\
\text { Sentido común, } \\
\text { imaginación. } \\
\text { Memoria. } \\
\text { Cogitativa }\end{array}$ & $\begin{array}{l}\text { Intelecto: } \\
\text { Concepto } \\
\text { Juicio } \\
\text { Raciocinio }\end{array}$ & La verdad \\
\hline
\end{tabular}

${ }^{18}$ IMBERNON, F., La educación en el siglo XXI., p.177.

${ }^{19}$ Valores trascendentes: verdad, bien, belleza, unidad.

${ }^{20}$ Egociudadano, egoísta, el sí y para sí mismo.

Ecociudadano, ecosistema, ecología, ecoética, ecocivismo., vid., MORIN, E., La Complexité humaine., Ed. Flammarión., Paris., 1998., p.380.

${ }^{21}$ Vid., BURON, J. Enseñar a aprender.

\section{BIBLIOGRAFÍA}

BURON, Javier., Enseñara aprender: Introducción a la metacognición., Ed. Mensajero., España., 1999., 157 p.

IMBERNON, F., La educación en el SigloXXI. Losretosdel futuro inmediato., Ed. GRAÓ., Barcelona., 1999., 180 p.

MARGALT, Avishai., La sociedad decente., Ed. Paidós., Barcelona., 1997., $222 \mathrm{p}$. 
MONEREO, C., etal. Profesoresy alumnos estratégicos., Pasial., España., 1993., $214 \mathrm{p}$.

MORIN, Edgar., La complexité humaine., Flammarion., Panis., 1998., 380 p. MORIN, Edgar., La téte bien faite., Ed. Seuil., Paris., 1999., 153 p.

MORIN, Edgar., Lesseptsavoirsnecessairesal'education du futur., Ed. Seuil., Paris., 2000., 129 p.

NAVAL, Concepción., «a autonomía: đin último de la educación moral?’en Lengua, Literatura y Valores., Newbook Ediciones., España., 1998., p.141-154.

NAVAL, Concepción., et al. «Es posible una continuidad entre hombre y sociedad?»en Revista Española dePedagogía., año LVIII., mayo-agosto 2000., No.216.

PELAEZ, Miguel Ángel., Ética, profesión y virtud., Rialp., España., 1991., $129 \mathrm{p}$.

QUINTANA CABANAS, José Maná., Pedagogía Axiológica. La educación antelosvalores., Dykinson., Madrid., 1998., 438 p.

ROUSSEAU, Jean-Jacques., Emileou del'Education., Flammarion., Paris., 1966., $629 \mathrm{p}$.

UNESCO. Les clés du XXIe siécle., Préface de Koîchiro MATSUURA, directeur géneral de l'UNESCO., Ed. Seuil., Paris., 2000., 515 p. 